\title{
Osteonecrosis after treatment for heterotopic ossification in spinal cord injury with the combination of surgery, irradiation, and an NSAID
}

\author{
AA van Kuijk ${ }^{*}, 1$, HJM van Kuppevelt ${ }^{1}$ and DB van der Schaaf ${ }^{2}$ \\ ${ }^{1}$ Department of Rehabilitation, Sint Maartenskliniek Nijmegen, The Netherlands; ${ }^{2}$ Department of Orthopaedic \\ Surgery, Sint Maartenskliniek Nijmegen, The Netherlands
}

\begin{abstract}
Study design: Case report.
Objective: Heterotopic ossification (HO) is a frequent complication in spinal cord injury (SCI) that is often difficult to treat. Although surgery may become necessary, operative resection has been associated with complications and poor outcome due to a high recurrence rate. Additional methods of treatment to reduce the recurrence rate have been developed, including post operative irradiation and NSAIDs. This article presents three patients, who developed an osteonecrosis of the femoral head after the combined treatment for $\mathrm{HO}$ of surgery, irradiation, and an NSAID.
\end{abstract}

Spinal Cord (2000) 38, 319-324

Keywords: heterotopic ossification; spinal cord injury; surgery; irradiation; osteonecrosis

\section{Introduction}

Heterotopic ossification ( $\mathrm{HO})$ is a frequent complication in spinal cord injury (SCI). The incidence in literature ranges from $10 \%$ to $53 \%$ depending on study design, the methods of detection, and the awareness of the disease. ${ }^{1-8}$ In $20 \%$ to $30 \%$ of spinal cord injured patients, clinically significant HO develops, most frequently at the hip. ${ }^{5,8-15}$ Reduction of hip joint movement may lead to loss of an adequate sitting position, pressure sores, increase of spasticity, and pain. ${ }^{9}$ These problems often require surgical resection of HO. However, operative resection of $\mathrm{HO}$ is often associated with severe complications and poor outcome. Complications include deep or superficial infection, post-operative haemorrhage, fractures, and a considerable recurrence rate. Different prophylactic treatment methods have been developed to reduce the recurrence rate, including diphosphonates, nonsteroidal antiinflammatory drugs (NSAID), and more recently irradiation. All methods seem to be successful in preventing $\mathrm{HO}$ after (elective) total hip arthroplasty. To date, no controlled studies are available about the effectiveness of these methods in spinal cord injured patients. We report three cases of osteonecrosis of the femoral head after surgical resection of heterotopic ossification with prophylactic post operative irradiation and an NSAID.

\section{Case reports}

A 30-year-old man sustained a C6 fracture resulting in C6-complete tetraplegia (ASIA A impairment scale)

*Correspondence: AA van Kuijk, Sint Maartenskliniek, Department of Rehabilitation, PO BOX 9011, 6500 GM Nijmegen, The Netherlands following a motor-vehicle accident in October 1993. Ten months post injury, he developed a deep vein thrombosis in the iliac and femoral vein of the left leg, complicated by pulmonary embolism. Anti-coagulation therapy was complicated by retroperitoneal bleeding, and computerised tomography of the abdomen showed migration of blood into the quadriceps region of the left leg. Despite treatment, the left thigh remained red and swollen. Laboratory evaluation showed an increased serum alkaline phosphatase level, and the anteroposterior roentgenogram of the pelvis revealed a calcification in the quadriceps region. $\mathrm{HO}$ was diagnosed, and treatment with sodium etidronate $10 \mathrm{mg} / \mathrm{kg} /$ day was started for 12 weeks. The evolution of $\mathrm{HO}$ was monitored by serial anteroposterior roentgenograms of the pelvis and alkaline phosphatase levels. On serial roentgenograms an increase in $\mathrm{HO}$ was seen until January 1997. Alkaline phosphatase levels remained elevated until June 1997. Bone scans in December 1994 and April 1995, showed increased uptake of the HO. Range of motion of the hip joint decreased over time and 17 months after injury the hip became ankylotic with the resulting loss of an adequate sitting position. A technetium bone scan at that time showed no increased uptake, and the HO of the left hip was resected in June 1997. Preoperatively, a 3-D computerised tomography was made to define the exact configuration and extent of $\mathrm{HO}$ and its relationship to surrounding structures. ${ }^{9,16}$ Measurements of ROM in flexion and extension direction were made pre and post-operatively (Table 1). Surgery was performed under fluoroscopic control using a ventrolateral approach. Resection was performed until $90^{\circ}$ of hip flexion could be obtained. Post-operative management 
Table 1 Range of motion (ROM)

\begin{tabular}{lccc}
\hline ROM & Patient 1 & Patient 2 & Patient 3 \\
\hline HO diagnosis & flex/ext $100^{\circ}-0^{\circ}-0^{\circ}$ & flex/ext $95^{\circ}-0^{\circ}-10^{\circ}$ & flex $/$ ext $90^{\circ}-0^{\circ}-0^{\circ}$ \\
Pre-operative & ankylosis in $8-^{\circ}$ flexion & ankylosis in $80^{\circ}$ flexion & $\begin{array}{c}\text { ankylosis in } 90^{\circ} \text { flexion } \\
\text { flex } / \text { ext } 110^{\circ}-0^{\circ}-0^{\circ}\end{array}$ \\
Post-operative & flex/ext $100^{\circ}-0^{\circ}-0^{\circ}$ & flex $/$ ext $100^{\circ}-0^{\circ}-10^{\circ}$ & .
\end{tabular}

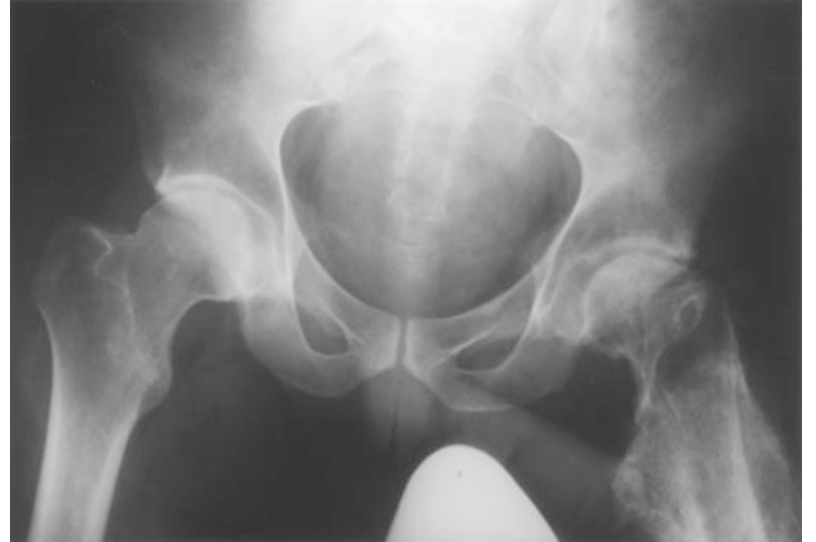

Figure 1 Postoperative AP of the pelvis demonstrating osteonecrosis of the right hip, with deformation of the collum femoris

included irradiation with $7 \mathrm{~Gy}$ in one session and indomethacin $25 \mathrm{mg}$ three times daily for 6 weeks. Follow up roentgenograms made respectively 1 week, 6 weeks, and 3 months post-operatively, revealed no signs of fractures, or of $\mathrm{HO}$ recurrence. Incidental osteonecrosis of the femoral head was first seen 3 months after operation (Figure 1). Two years after surgery, adequate sitting position was preserved, as was ROM, and no increase of spasm or of pain have been noted.

The second patient concerns a 31-year-old man with a T7 complete paraplegia (ASIA A impairment scale) due to transverse myelitis in May 1995. Four months after disease onset, laboratory evaluation revealed an acute rise of the serum alkaline phosphatase level. Physical examination of the hip joint showed decreased range of motion. In addition, an anteroposterior roentgenogram of the pelvis indicated $\mathrm{HO}$ at the level of the great trochanter and the medial part of the femoral neck on the left side. Despite treatment with sodium etidronate $(20 \mathrm{mg} / \mathrm{kg} /$ day for the first 2 weeks and $10 \mathrm{mg} / \mathrm{kg} /$ day for an additional 10 weeks), range of motion continued to decrease. As a result of the inadequate sitting position and increase in spasticity, a decubitus wound in the region of the left tuber ischiadicum developed. Alkaline phosphatase levels remained elevated until February 1997. By December 1997 the hip joint became clinically ankylotic and a corresponding technetium bone scan showed no increased uptake (Figure 2). A 3D-computerised

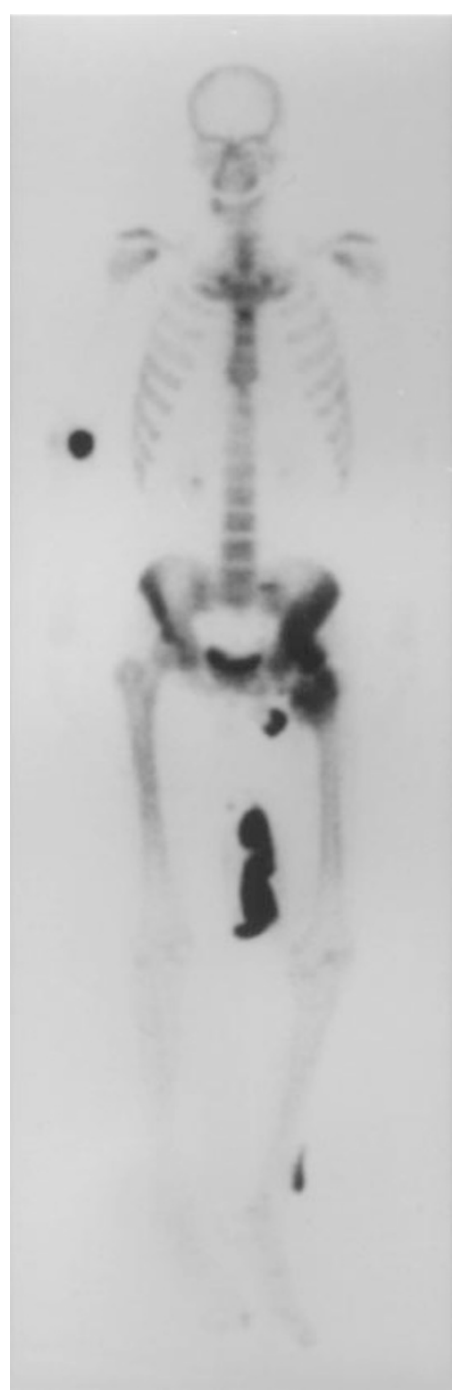

Figure 2 99m Technetium bone scan demonstrating heterotopic ossification about the left hip, os ileum and femur

tomography was made (Figure 3), and surgical resection of $\mathrm{HO}$ of the hip followed in February 1998. An identical operative and post-operative regime as described above for the first patient was used. Two months after operation, fever developed with an increase of spasm, and the patient was admitted to the hospital. A deep infection of the hip with osteonecrosis of the femoral head was diagnosed. No 


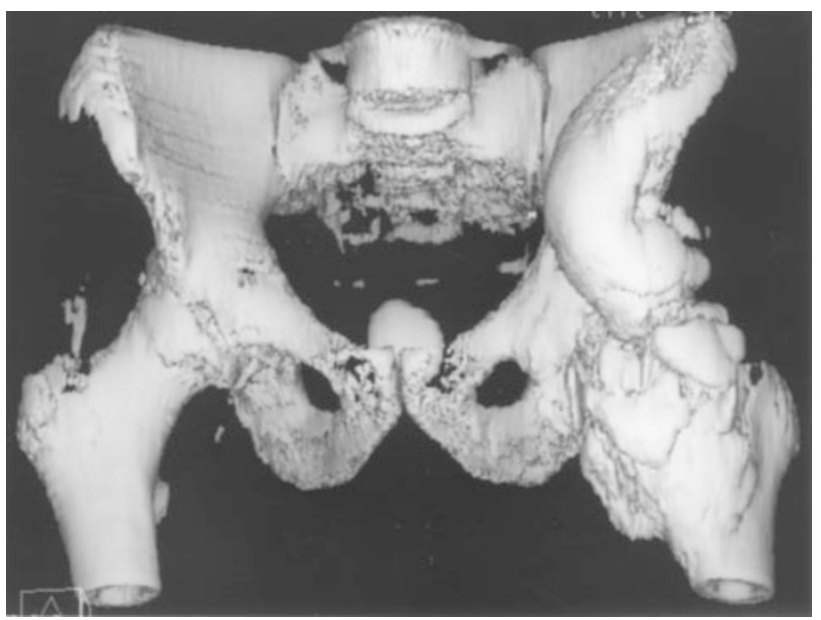

Figure 3 3D-computerised tomography demonstrating the extent and configuration of the heterotopic ossification

signs of fracture were seen on follow up roentgenograms after surgery. Follow up until 1 year postoperative revealed no signs of $\mathrm{HO}$ recurrence, and an adequate sitting position as well as ROM, have been preserved.

The third patient presented concerns a 21-year-old man with T7 complete paraplegia (ASIA A impairment scale) due to a gunshot wound in December 1994. Two months after SCI, at the admission to our rehabilitation centre, a decreased range of motion of both hips was discovered on physical examination. Serum alkaline phosphatase was elevated, and the anteroposterior roentgenogram of the pelvis showed $\mathrm{HO}$ on both hips. Sodium etidronate $(20 \mathrm{mg} / \mathrm{kg} / \mathrm{day}$ for the first 2 weeks and $10 \mathrm{mg} / \mathrm{kg} /$ day for an additional 10 weeks) was given. Technetium bone scans showed increased uptake until May 1997, at that time a steady state in the ratio of uptake between normal and heterotopic bone was seen. In January 1998 the bone scan revealed again a steady state and the right hip was operated on $\mathrm{HO} 3$ years after diagnosis. The patient underwent an identical operative and postoperative regime as the first two patients presented. Three months after operation, osteonecrosis of the femoral head was discovered on follow up anteroposterior roentgenogram of the pelvis. There were no signs of fracture or of $\mathrm{HO}$ recurrence, and an adequate sitting position was preserved, as was ROM.

\section{Discussion}

$\mathrm{HO}$, a frequent complication in SCI, develops in $10 \%$ to $53 \%$ of all SCI patients. In $20 \%$, HO leads to reduction in range of motion leading to loss of function, and in 5\% ankylosis develops. ${ }^{1-7,9-12,14}$ HO develops below the level of injury, most commonly at the hip $8,10-15,17$ and usually along the anteromedial plane of the hip joint. ${ }^{5,9,11}$ Complete transverse SCI is more commonly associated with $\mathrm{HO}$ than incomplete lesions. ${ }^{1,8,14,17}$ Other variables associated with $\mathrm{HO}$ formation are the presence of pressure sores and spasticity. ${ }^{9}$ Although it can develop even after several years, $\mathrm{HO}$ is generally diagnosed between 1 to 4 months post-injury with a peak incidence at 2 months. $1,6,18$

The most common finding on clinical examination to indicate the presence of $\mathrm{HO}$ is a decreased range of motion, followed by a localised swelling. ${ }^{9,19}$ Erythema and warmth also occur. The early symptoms must be differentiated from osteoarthritis, thrombophlebitis, and tumour. An elevated serum alkaline phosphatase may be of value in differentiating early $\mathrm{HO}$ from other inflammations, as elevation of alkaline phosphatase levels precede the roentgenographic diagnosis. The usual interval is reported less than two weeks, but an interval of 4 months has been reported. ${ }^{7}$ Thus in the early stage of $\mathrm{HO}$ formation, the roentgenogram may be negative. Ultrasonography can be used in the early identification of clinically suspected $\mathrm{HO}$ and to differentiate $\mathrm{HO}$ from deep vein thrombosis, or a developing pressure sore, infection, or tumour. ${ }^{20-22}$

In the literature the evolution of $\mathrm{HO}$ is monitored by serial anteroposterior roentgenograms of the pelvis, by serum alkaline phosphatase levels, and/or by bone scintigrams. Although alkaline phosphatase levels are indices for active bone formation, normal levels do not predict maturity or recurrence, and alkaline phosphatase levels do not correlate with either peak activity or number of $\mathrm{HO}$ lesions. ${ }^{6,9,23}$ However, the roentgenographic appearance is not a reliable parameter for judging the maturity of $\mathrm{HO},{ }^{7,11}$ and it is an insensitive tool for predicting recurrence. ${ }^{23}$ With a large block of ectopic bone interpretation of the roentgenogram is difficult, and immature elements may be obscured by mature bone. ${ }^{11}$ The $99 \mathrm{~m}$ technetium bone scan is the most sensitive tool in identifying $\mathrm{HO}$ and determining maturity. ${ }^{23,24}$ Serial decrease or a steady-state uptake ratio between normal and heterotopic bone is a reliable indicator for $\mathrm{HO}$ maturity. ${ }^{9}$ In the literature the occurrence of maturity of $\mathrm{HO}$ in SCI is reported at $12-18$ months after first clinical presentation. $5,10,18$

To date there is no satisfactory treatment to prevent $\mathrm{HO}$ occurrence. Once HO has been documented, patients can be treated with sodium etidronate, which to some extent has an inhibitory effect on the development of $\mathrm{HO}$ but which does not inhibit the formation of osteoied matrix. After diphosphonate treatment has been discontinued, $\mathrm{HO}$ continues to progress; the extent of recurrence varies in literature. $4,14,25-27$ Stover reported disodium to be potentially effective in preventing $\mathrm{HO}$ provided treatment was started before HO developed. The drug had minimal influence on $\mathrm{HO}$ already present, although the rate of progression may possibly have been slowed. $^{26}$ NSAIDs are used after total hip arthroplasty to prevent $\mathrm{HO}$ occurrence. ${ }^{27,28}$ The effect of NSAID has been attributed to inhibition of the inflammatory response or suppression of mesenchymal cell proliferation. ${ }^{9}$ 
The indication for surgery is improvement of hip motion to achieve adequate sitting and/or standing position, reduction of spasms and prevention of pressure sores. ${ }^{11,29,30}$ In the literature there is no consensus as to the most effective timing for the surgery. It has been suggested that recurrence of $\mathrm{HO}$ after resection is lessened if $\mathrm{HO}$ is removed during a radionuclide steady state. ${ }^{9-11,24,31}$ While Stover did not find any correlation between the extent of recurrence and length of onset of $\mathrm{HO}$ to surgery, ${ }^{29}$ Freebourn stated that early resection of immature $\mathrm{HO}$ may not be predictive of a higher recurrence rate. ${ }^{32}$

Because $\mathrm{HO}$ resection is associated with poor outcome mainly due to a high recurrence rate, different prophylactic methods have been developed to reduce the recurrence rate. Both irradiation and indomethacin seem to be effective in preventing $\mathrm{HO}$ occurrence after total hip replacement. ${ }^{27,28,33-36}$ However, the exact mechanism by which irradiation prevents HO development remains unknown. Irradiation may disrupt the differentiation process of pluripotential mesenchymal cells into osteoblasts. ${ }^{32,34}$ It may also serve to decrease pain perception associated with the tissue inflammatory reaction around the side of $\mathrm{HO}$, or it may be involved in ablation of pain receptors. ${ }^{34}$ Post-operative irradiation has been reported to decrease the recurrence of $\mathrm{HO}$ after surgical resection of $\mathrm{HO}$ in SCI, but no controlled studies are available. ${ }^{37-40}$ Sautter-Bihl et al treated 20 SCI patients with irradiation with $10 \mathrm{~Gy}$ in single fractions of 2 to $2.5 \mathrm{~Gy}$. In 15 patients irradiation was performed as primary treatment to prevent HO formation, in seven patients it was used after operative $\mathrm{HO}$ resection to prevent $\mathrm{HO}$ recurrence. Four patients of the seven operated on, received a total hip prosthesis. In a follow up period of 12 weeks, none of the patients showed progression of HO. In five patients ROM and sitting position had decreased after 44 months. No radiographic control of the affected hip was performed. ${ }^{38}$

Reported complications of radiation therapy include delayed wound and bone healing as well as the risk of developing a radiation induced sarcoma. The latter complication is not mentioned at radiation doses less than 30 Gy delivered within a 3-week period, ${ }^{41,42}$ however it limits the use of radiation therapy as the primary prevention of $\mathrm{HO}$, when the patient's long life expectancy and the frequent involvement of multiple joints in spinal cord injured patients are taken into account. ${ }^{39}$

Anti-inflammatory drugs inhibit the formation of prostaglandins and related substances which trigger the local bone remodelling, thereby reducing the formative and resorptive phases of bone remodelling. ${ }^{27,28,32}$ Both indomethacin and radiation therapy could exert their prophylactic effects by preventing the differentiation of the pluripotential mesenchymal cells into osteogenic cells.

Resection of $\mathrm{HO}$ is associated with severe complications, including excessive bleeding, deep or superficial (wound) infection and fractures. In our series, all patients developed an osteonecrosis, a complication after surgery not previously mentioned in literature. While the exact aetiology and pathogenesis of osteonecrosis are still unknown, several conditions have been associated with osteonecrosis. These include fractures and dislocations of the hip, therapeutic irradiation, corticosteroid therapy, and the chronic use of anti-inflammatory as well as analgesic preparations. ${ }^{43,44}$

Osteonecrosis is a well known, but infrequent complication of therapeutic pelvic irradiation. In the literature, two theories for the pathogenesis of radiation induced osteonecrosis exist. In the first theory, irradiated bone is proposed to be more susceptible to infection and trauma resulting from radiation induced vascular damage. After irradiation, fibrosis and intimal thickening of the small arterioles with luminal narrowing and obliteration of vessels in bone, synovium and surrounding capsular tissues have been reported. ${ }^{45-48}$ However, since the degree of this vascular impairment is insufficient to produce osteonecrosis, other factors such as trauma or infection must co-exist. ${ }^{43}$

Another proposed mechanism for radiation induced osteonecrosis is the destruction of the bone cell population. After therapeutic irradiation, the most prominent change in bone is osteoporosis with histological evidence for decreased osteoblast and osteoclast activity, leading to disruption of the normal equilibrium of bone metabolism. ${ }^{45,48}$ However irradiation does not completely nor permanently suppress the osteoblastic activity. Hence, one must conclude that a combination of cell population destruction with other predisposing factors leads to osteonecrosis. ${ }^{45,48}$

Furthermore, osteoporosis, in conjunction with the diminution of the normal and protective pain responses, could lead to osteonecrosis. In spinal cord injured patients, disuse osteoporosis is frequently observed below the level of SCI, and this is more pronounced at the hip. ${ }^{49}$ Microfractures of subchondral bone occur in osteoporosis, which is more likely in states of diminished sensibility in particular if there is increased exposure to trauma. ${ }^{43}$ Such microfractures could lead to cell death, and the subsequent subarticular collapse of the femoral head. In addition enzymatic products of cell death might induce a local inflammatory exudate, and thus mediate a progressive increase in pressure within a closed bony compartment. Such bone marrow pressure may directly affect local vascular resistance and bloodflow. ${ }^{44}$ The end result of the above process is that the increased bone marrow pressure could impede the blood supply, leading to osteonecrosis.

In this study a combination of treatments were used: an operation, followed by irradiation, and the use of an anti-inflammatory drug. Although radiation was performed in one session and in a relatively minor dose compared to therapeutic radiation therapy, the combination of irradiation and the suspected pre- 
existing osteoporosis may have led to osteonecrosis. It is also possible that during the operation damage to the vessels of the vascular system of the femur might have occurred. As mentioned above, such damage in bloodflow could lead to increased bone marrow pressure and predispose to osteonecrosis. However, the exact cause of osteonecrosis in our patients remains unknown.

Despite the osteonecrosis, adequate sitting position, which was the main reason to operate, was preserved, as was the range of motion of the hip joint. Up until now, the osteonecrosis does not seem to have produced any functional implications. Obviously the relatively short follow up period makes it impossible to predict any future implications of osteonecrosis.

In conclusion, although irradiation is known to be effective in the prevention of $\mathrm{HO}$ after total hip surgery, the three cases of femoral head osteonecrosis following a combined treatment of surgery, irradiation and an NSAID indicates that such a regime may not be appropriate for SCI patients. From our study the clinical implications of osteonecrosis appear minimal but are not completely understood at this time.

\section{References}

1 Bravo-Payno $\mathrm{P}$ et al. Incidence and risk factors in the appearance of heterotopic ossification in spinal cord injury. Paraplegia 1992; 30: $740-745$

2 Collates SC, Clinch DM, Viennese MD. Neuromuscular complications of heterotopic ossification following spinal cord injury. Paraplegia 1993; 31: $51-57$.

3 Damanski M. Heterotopic ossification in paraplegia. J Bone Joint Surg 1961; 43B: $286-299$.

4 Garland DE, Alday B, Venos KG, Vogt JC. Diphosphonate treatment for heterotopic ossification in spinal cord injury patients. Clin Orthop 1983; 176: $197-200$

5 Garland DE. Clinical observations on fracture and heterotopic ossification in the spinal cord and brain injured populations. Clin Orthop 1988; 233: 86-101.

6 Hsu J, Sakimura I, Stauffer E. Heterotopic ossification around the hip joint in spinal cord injured patients. Clin Orthop 1975; 112: $165-169$.

7 Tibone J, Sakimura I, Nickel VL, Hsu JD. Heterotopic Ossification around the hip in spinal cord-injured patients. A long term follow-up study. J Bone Joint Surg 1978; 60A: $769-$ 775 .

8 Wittenberg RH, Peschke U, Bötel U. Heterotopic ossification after spinal cord injury; epidemiology and risk factors. $J$ Bone Joint Surg 1992; 74B: 215-218.

9 Garland DE. A clinical perspective on common forms of acquired heterotopic ossification. Clin Orthop 1991; 263: 13-29.

10 Wharton G, Morgan T. Ankylosis in the paralysed patient. $J$ Bone Joint Sug 1970; 52A: 105-112.

11 Wharton G. Heterotopic ossification. Clin Orthop 1975; 112: $142-149$.

12 Stover S, Hataway C, Zeiger H. Heterotopic ossification in spinal cord injured patients. Arch Phys Med Rehabil 1975; 56: 199-204.

13 Venier LH, Ditunno JF. Heterotopic ossification in the paraplegic patient. Arch Phys Med Rehabil 1979; 52: 475-479.

14 Hernandez A et al. The pararticular ossification's on our paraplegics and tetraplegics: a survye of 704 patients. Paraplegia 1978; 16: $272-275$.

15 Chantraine A, Minaire P. Para-osteo-arthropathies. Scand J Rehabil 1981; 13: $31-37$.

16 Béthoux $\mathrm{F}$ et al. Heterotopic ossification and rhabdomyolysis. Paraplegia 1995; 33: $164-166$.
17 Lal S, Hamilton BB, Heinemann A, Betts HB. Risk factors for heterotopic ossification in spinal cord injury. Arch Phys Med Rehabil 1989; 70: $387-390$.

18 Hardy A, Dickson J. Pathological ossification in traumatic paraplegia. J Bone Joint Surg 1963; 45B: 76-87.

19 Dejerine A, Ceillier MA. Para-ostéo-arthropathies des paraplégiques par lésion médullaire: Étude clinique et radiographique. Ann Méd 1918; 5: 497-535.

20 Snoecx M, De Muynck M, Van Laere M. Association betwwen muscle trauma and heterotopic ossification in spinal cord injured patients. Reflections on causal relationships and diagnostic value of ultrasonography. Paraplegia 1995; 33: 464-468.

21 Cassar-Pullincino VN et al. Sonographic diagnosis of heterotopic bone formation in spinal injury patients. Paraplegia 1993; 31: $40-50$.

22 Bodley R, Jamous A, Short D. Ultrasound in the early diagnosis of heterotopic ossification in patients with spinal injuries. Paraplegia 1993; 31: 500-506.

23 Garland DE, Orwin JF. Resection of heterotpic ossification in patients with spinal cord injuries. Clin Orthop 1991; 263: 87-93.

24 Freed JH, Hahn H, Menter R, Dillon T. The use of three phase bone scan in the early diagnosis of heterotopic ossification and in the evaluation of Didronel therapy. Paraplegia 1982; 20: $208-$ 216.

25 Banovac K, Gonzalez F, Wade N, Bowker JJ. Intravenous disodium etidronate therapy in spinal cord injury patients with heterotopic ossification. Paraplegia 1993; 31: 660-666.

26 Stover SL, Niemann KMW, Miller III JM. Disodium Etidronate in the prevention of postoperative recurrence of heterotopic ossification in spinal cord injury patients. J Bone Joint Surg 1976; 58A: $683-688$.

27 Kjaersgaard-Andersen P, Schmidt SA. Total hip arthroplastythe role of anti-inflammatory medications in the prevention of heterotopic ossification. Acta Othop Scand 1990; 263: $78-86$.

28 Kjaersgaard-Andersen $\mathrm{P}$ et al. Indomethacin for prevention of heterotopic ossification. Acta Orthop Scand 1993; 64: 639-642.

29 Stover SL, Niemann KMW, Tulos J. Experience with surgical resection of heterotopic bone in spinal cord injury patients. Clin Orthop 1991; 263: $71-77$.

30 Aanholt van PCTh, Martina JD, Eisma WHE. Ectopische botvorming, diagnostiek en behandeling. $N T v G$ 1991; 9: 380 384.

31 Muheim G, Donath A, Rossier AB. Serial scintigrams in the course of ectopic bone formation in paraplegic patients. $\mathrm{Am} \mathrm{J}$ Roentgenol 1973; 118: 865 -869.

32 Freebourn TM, Barber DB, Able AC. The treatment of immature heterotopic ossification in spinal cord injury with the combination surgery, radiation therapy and NSAID. Spinal Cord 1999; 37: $50-53$.

33 Pelligrini VD, Gregoritch SJ. Preoperative irradiation for prevention of heterotopic ossification. J Bone Joint Surg 1996; 78A: $870-881$.

34 Schaeffer MA, Sosner J. Heterotopic ossification: Treatment of established bone with radiation therapy. Arch Phys Med Rehabil 1995; 76: $284-286$.

35 Ayers DC, Pelligrini VD, Evarts CM. Prevention of heterotopic ossification in high-risk patients by radiation therapy. Clin Orthop 1991; 263: 87-93.

36 Biering-Sørensen F, Tønedenvold E. Indomethacin and disodium etidronate for the prevention of recurrence of heterotopic ossification after surgical resection. Paraplegia 1993; 31: $513-$ 551 .

37 Meiners T, Abel R, Bõhm V, Gerner HJ. Resection of heterotopic ossification of the hip in spinal cord injured patients. Spinal Cord 1997; 35: $443-445$.

38 Sautter-Bihl ML et al. The radiotherapy of heterotopic ossification in paraplegics. Strahlentherapie und Onkologie 1995; 171: $454-459$

39 McAuliffe JA, Wolfson AH. Early excision of heterotopic ossification about the elbow followed by radiation therapy. $J$ Bone Joint Surg 1997; 79B: $749-755$ 
40 Ellerin BE et al. Current therapy in the management of heterotopic ossification of the elbow: a review with case studies. Am J Phys Med Rehabil 1999; 78: 259-271.

41 Brady LW. Radiation induced sarcomas of bone. Skel Radiol 1972; 4: $72-78$.

$42 \mathrm{Kim} \mathrm{JH}$ et al. Radiation induced soft tissue and bone sarcoma. Radiology 1978; 129: 501 - 508.

43 Solomon L. Drug-induced arthropathy and necrosis of the femoral head. J Bone Joint Surg 1973; 55B: 246-261.

44 Cruess RL. Osteonecrosis of bone. Current concepts as to aetiology and pathogenesis. Clin Orthop 1986; 208: $30-39$.

45 Csuka M, Brewer BJ, Lynch KL, McCarty DJ. Osteonecrosis, fractures, and protrusio acetabuli secondary to X-irradiation therapy for prostatic carcinoma. J Rheumatol 1987; 14: 165-170.
46 Hasselbacher P, Schumacher HR. Bilateral protrusio acetabuli following pelvic irradiation. J Rheumatol 1977; 4: 189-196.

47 Delaere $\mathrm{O}$ et al. Long-term sequelae of pelvis irradiation: Histological and microradiographical study of a femoral head. Clin Rheumat 1991; 10: 206-210.

48 Henry AP, Lachmann E, Tunkel RS, Nagler W. Pelvic insufficiency fractures after irradiation: Diagnosis, management, and rehabilitation. Arch Phys Med Rehabil 1996; 77: $414-416$.

49 Jaovisidha S et al. Influence of heterotopic ossification of the hip on bone desitometry: a study in spinal cord injured patients. Spinal Cord 1998; 36: $647-653$. 